\title{
Advances in motion control of gas pipeline detection robot
}

\author{
SHI Yan, MU ZhenXin, CAI MaoLin, SONG Hua \& WANG YiXuan* \\ School of Automation Science and Electrical Engineering, Beihang University, Beijing 100191, China
}

Received February 21, 2019; accepted August 23, 2019; published online September 24, 2019

Citation: Shi Y, Mu Z X, Cai M L, et al. Advances in motion control of gas pipeline detection robot. Sci China Tech Sci, 2020, 63: 877-878, https://doi.org/ $10.1007 / \mathrm{s} 11431-019-1435-2$

Pipelines are important means of oil and gas transportation, which are widely used in chemical, electric power and municipal engineering. However, the pipeline may be cracked or broken because of factors such as corrosion of the transmission medium and natural disasters. The traditional pipeline inspection is done manually, with long inspection time and large workload. In this case, people invented the pipeline inspection robot, which greatly improved the detection efficiency [1-3]. Pipeline detection is divided into internal detection and external detection, while oil and gas pipeline detection robot is mainly for internal detection.

Oil and gas pipeline inspection robots, which are widely used in the detection of oil and gas pipelines, can walk in pipelines and complete accurate inspection tasks. However, the control problem of the movement of the oil and gas pipeline detection robot in the pipeline is the primary problem, which is divided into two parts: motion control and attitude control [4]. Under the influence of frictional resistance and fluid medium resistance, the movement of the robot in the closed pipeline will have speed fluctuations, and the motion posture cannot be controlled autonomously at the bend of the pipeline, which is easy to get stuck in the pipeline and affect the accuracy of the sensor mounted on the detection robot, and even cause damage to the sensor.

At present, there are two kinds of speed control methods for oil and gas pipeline detection robots. The first method is fluid-driven control, which adjusts the pressure difference between the front and back ends by controlling the opening of the throttle hole on the robot through the speed motor, thus

\footnotetext{
*Corresponding author (email: yesoyou@gmail.com)
}

realizing the control of the forward speed of the pipeline robot [5]. The speed control process is shown in Figure 1. The second is motor-driven control, which directly controls the speed of a wheeled or tracked pipeline robot through a DC motor [6,7].

The attitude control of oil and gas pipeline detection robots mainly has the following two methods. One is a visual servobased pipeline following scheme, which obtains the required velocity and position information by CCD camera, processes the obtained information, and realizes speed control and attitude adjustment through the inner and outer loop controllers [8]. However, this solution is limited to environments with good visual conditions and cannot work in harsh environments.

The second way is a new structural design in which the wheel of the pipeline robot has a certain wheel inclination that makes the robot climb vertically along the pipeline. Because of the flexible mechanism on the wheel bracket, the robot has good pipeline adaptability [9]. This scheme has the advantage of automatic attitude adjustment and simplifies the attitude control of pipeline robot in pipeline. However, it is only based on the model development of UGNX software, which is still in the initial experimental test.

At present, there is no better speed control scheme. Although the fluid drive control system is simple and can use the energy of the fluid medium for long-distance operation, the stability of the control system is poor due to the fluctuation of pressure in the pipeline and the change of friction force between the driving bowl and the pipeline, which makes the control system vulnerable to the influence of fluid. The advantage of the motor drive control scheme is that it is 


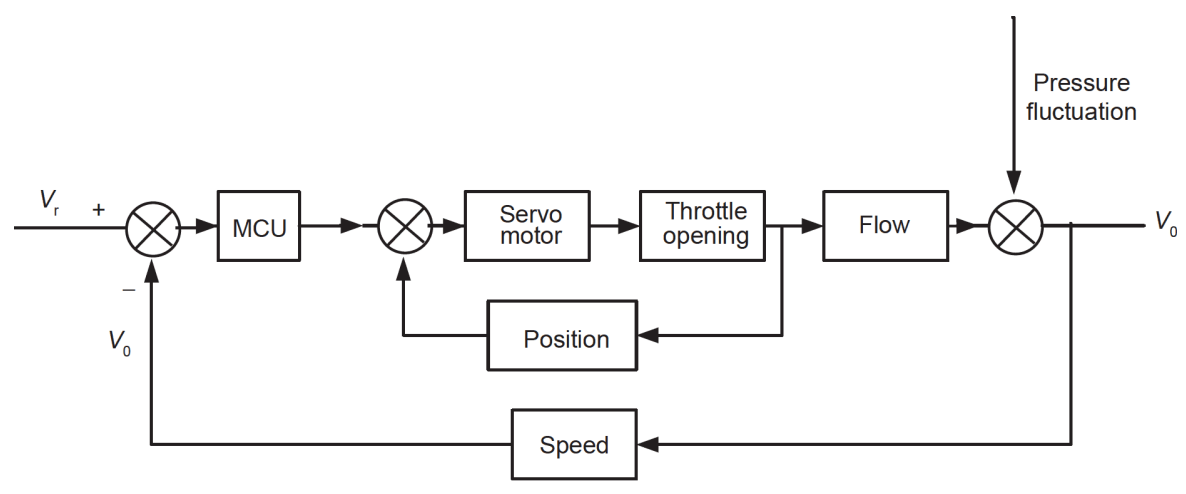

Figure 1 Differential pressure pipeline inspection robot speed control process.

easy to control the speed and less affected by the environment. However, its mechanical structure is complex, pipeline adaptability is poor, it is greatly affected by friction, and it is difficult to run for long distance because of the influence of energy supply.

In the future, it is of great importance that the research on velocity control method of pipeline robot. The main problems of motion control of gas pipeline inspection robot are energy supply and speed detection. The future development direction of gas pipeline inspection robot is to solve the problem of energy supply through fluid drive. For the speed control of the gas pipeline inspection robot, the main research goal in the future is how to solve the response delay of the actuator and increase the anti-interference of the system, and how to apply the control algorithm to the actual speed control system.

This work was supported by the National Natural Science Foundation of China (Grant No. 51975024).
1 Sun L, Chen C, Sun Q Q. Experimental and finite element analyses on the corrosion of underground pipelines. Sci China Tech Sci, 2015, 58: 1015-1020

2 Ibrahimov B, Namazov M. Robotics in petroleum and safety requirements forcing Open Innovation to be embraced. IFAC-PapersOnLine, 2018, 51: 688-692

3 Shi Y, Hao L, Cai M, et al. High-precision diameter detector and three-dimensional reconstruction method for oil and gas pipelines. $\mathrm{J}$ Pet Sci Eng, 2018, 165: 842-849

4 Nee L V, Elamvazuthi I, Ganesan T, et al. Development of a laboratory-scale pipeline inspection robot. Procedia Comput Sci, 2015, 76: 9-14

5 Mirshamsi M, Rafeeyan M. Dynamic analysis and simulation of long pig in gas pipeline. J Nat Gas Sci Eng, 2015, 23: 294-303

6 Qu Y, Durdevic P, Yang Z. Smart-spider: Autonomous self-driven inline robot for versatile pipeline inspection. IFAC-PapersOnLine, 2018, 51: $251-256$

7 Shukla A, Karki H. Application of robotics in onshore oil and gas industry-A review Part I. Robot Auton Syst, 2016, 75: 490-507

8 Allibert G, Hua M D, Krupínski S, et al. Pipeline following by visual servoing for Autonomous Underwater Vehicles. Control Eng Pract, 2019, 82: 151-160

9 Nayak A, Pradhan S K. Design of a new in-pipe inspection robot. Procedia Eng, 2014, 97: 2081-2091 